\title{
Path Integral Energy Landscapes for Water Dimer
}

\author{
Christophe L. Vaillant, Stuart C. Althorpe, and David J. Wales* \\ University Chemical Laboratories, Lensfield Road, Cambridge CB2 1EW, United Kingdom \\ E-mail: dw34@cam.ac.uk
}

\begin{abstract}
The energy landscape for a discretised path integral representation of the water dimer is characterized in terms of the localised (classical) and delocalised minima and transition states. The transition states are finite-temperature approximations to the exact instanton path, and are typically used to calculate the tunneling splittings or reaction rates. The features of the path integral landscape are explored, thus elucidating procedures that could usefully be automated when searching for instantons in larger systems. Our work not only clarifies the role of minima and transition states in path integral calculations, but also enables us to analyse the quantum-to-classical transition.
\end{abstract}

\section{Introduction}

Understanding the structure, dynamics and thermodynamics of water remains a key research goal, with applications ranging from biomolecular solvation to atmospheric chemistry. Building this understanding from the foundations provided by analysis of water clusters is an attractive approach, since smaller systems are usually more amenable to both high resolution 
experiments and accurate treatments of structure and dynamics in calculations. ${ }^{1-7}$ In par- $^{\text {- }}$ ticular, comparison of predicted tunneling splittings with experiment provides a demanding test of how well the intermolecular and intramolecular forces are represented over large amplitude motions, which explore configurations around both local minima and the transition states that connect them on the potential energy landscape. Here, the motion of light atoms subject to low barriers requires a proper account of quantum dynamical effects. ${ }^{8}$

Full quantum dynamics calculations are difficult due to the exponential scaling of the computational time with system size. One class of algorithms to overcome this exponential scaling relies on the path integral formulation of quantum mechanics, ${ }^{9}$ typically combined with either stochastic methods or approximations to the path integrals. Many such methods exist to treat both static ${ }^{10}$ and dynamic properties, including ring polymer molecular dynamics (RPMD), ${ }^{11-13}$ centroid molecular dynamics (CMD), ${ }^{14,15}$ Path integral Liouville dynamics, ${ }^{16,17}$ or the recently developed Matsubara dynamics. ${ }^{18,19}$ However, all these approaches have their own challenges, and the dynamics of liquid water have yet to be fully understood. $^{8,20-22}$

One practical approach that has been applied for quantum dynamics applications is the instanton approximation. ${ }^{23-26}$ This formulation results from applying the steepest-descent approximation (taking a second-order Taylor expansion about a stationary point and analytically integrating the resulting Gaussian functions) to the full path integral, and can be used to calculate molecular tunneling spectra, ${ }^{25-29}$ and the reaction rates of various chemical processes. ${ }^{23,30}$ The key component of any instanton calculation involves determining the minima and transition states that constitute the semiclassical path (known as the instanton), about which we apply the steepest-descent approximation. We note that an alternative path integral method exists for calculating tunneling spectra, which is in principle exact. ${ }^{31-33}$

Not all instanton paths yield contributions to the integrals that are relevant to the energy and time scales involved in the processes of interest. For example, in the water hexamer ${ }^{29}$ only two paths were found to contribute significantly to the tunneling splittings, despite 
the existence of around $10^{12}$ possible permutation-inversion (PI) operations that give rise to instantons. Until now, there has been no systematic method to characterise the landscape of possible instantons to determine the most relevant paths, with studies relying instead on ad hoc procedures (sometimes in tandem with experiment).

In the present contribution we aim to gain new insight into tunneling splitting dynamics by directly characterising the energy landscape for a ring polymer (i.e. a discretised path integral) representation of the water dimer. The energy landscape illustrates how the connections between transition states and local minima in the full ring polymer configuration space evolve with the number of polymer beads and with temperature, thus exploring the transition between quantum and classical regimes. The landscapes clearly show how the delocalised quantum paths that contribute to the quantum dynamics are related to the classical paths as the temperature rises. We also characterise the eigenvectors of zero and negative eigenvalue modes, which provide a helpful geometric understanding of the ring polymer motion. Our analysis paves the way for efficient automation of the process of calculating tunneling splittings, without the need for a priori knowledge of the dominant instanton paths.

\section{Methods}

All the methodology employed in the present work for exploring the energy landscape and constructing kinetic transition networks ${ }^{34-36}$ is well established, and has been described in

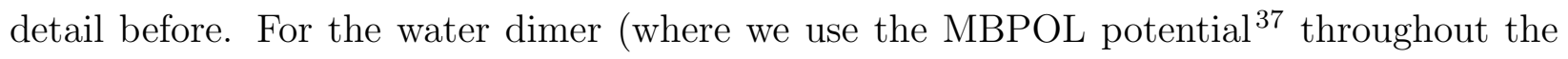
discussion) there are only eight distinct permutation-inversion isomers of the classical local minimum structure, ${ }^{38}$ and the resulting tunneling splitting pattern has been analysed in detail. ${ }^{39-46}$ These permutation-inversion isomers are structures that cannot be superimposed by an overall rotation, but differ only through the arrangement of atoms of the same element, which may be combined with inversion of all particle coordinates through the origin.

To characterise all the classical transition states corresponding to rearrangements of 
permutation-inversion isomers we attempt to connect all the pairs using the doubly-nudged ${ }^{47}$ elastic band ${ }^{48,49}$ (DNEB) approach, which provides transition state candidates for refinement using hybrid eigenvector-following. ${ }^{50-52}$ Here we define transition states geometrically, as saddle points of index one, with a single negative Hessian eigenvalue. ${ }^{53}$ The connectivity between minima was verified for each transition state by calculating approximate steepest-descent paths using the LBFGS approach, which is a limited memory version of the quasi-Newton Broyden, ${ }^{54}$ Fletcher, ${ }^{55}$ Goldfarb, ${ }^{56}$ Shanno, ${ }^{57}$ BFGS algorithm. As for kinetic transition networks based on classical minima and transition states, the ring polymer landscape can then be visualised using disconnectivity graphs, ${ }^{58,59}$ as discussed in the Results section.

The ring polymer landscape for a system with $N$ atoms and $P$ beads corresponds to $3 N P$ coordinates $\mathbf{X}=\left\{\mathbf{X}^{(1)}, \ldots, \mathbf{X}^{(P)}\right\}$, where $\mathbf{X}^{(\alpha)}$ is a $3 N$ dimensional vector defining the configuration for the water dimer (with $N=6$ ) in bead $\alpha$. The ring polymer energy landscape is determined by the potential function

$$
U_{P}=\sum_{\alpha=1}^{P}\left[\sum_{j=1}^{3 N} \frac{m_{j} P^{2}}{2 \beta^{2} \hbar^{2}}\left(X_{j}^{(\alpha)}-X_{j}^{(\alpha+1)}\right)^{2}+V\left(\mathbf{X}^{(\alpha)}\right)\right]
$$

where $\mathbf{X}^{(P+1)} \equiv \mathbf{X}^{(1)}$ for cyclic boundary conditions in the first term, which corresponds to $P$ images connected by harmonic springs. In (1), $\beta=1 / k_{\mathrm{B}} T$ is the inverse temperature, $V$ denotes the water dimer potential energy surface, and $m_{j}$ is the mass for the $j$ th degree of freedom. For finite values of $\beta$ and $P$ the instantons that are used to calculate tunneling splittings correspond to transition states of $U_{P}(\beta, \mathbf{X})$, with a single negative eigenvalue. This eigenvalue, $\lambda_{\text {neg }}$, tends to zero in the limit of low temperature, and it may be possible to characterise the instanton by minimisation if this optimisation is terminated before it collapses to a true minimum. ${ }^{60}$ However, in the present work, we focus on precise calculations of transition states, local minima, and pathways for finite values of $\beta$ and $P$, to gain insight into how the path integral landscape evolves for these parameters. The optimisation algorithms we apply are the same as for the classical potential energy surface, but applied to all 
$3 N P$ degrees of freedom.

The properties of the key stationary points can be elucidated by analysing the eigenvalues and eigenvectors of the second derivative matrix (the Hessian). For a mass-weighted Hessian this approach corresponds to normal mode analysis in the $3 N P$ dimensional space for a system of $N$ atoms represented by $P$ ring polymer images or beads. Since the eigenvalue spectrum, in terms of the number of positive, negative and zero eigenvalues, is not affected by mass weighting, ${ }^{61}$ we can also work with the Hessian directly, and this is the approach adopted here.

\section{Results}

\subsection{Pathways}

The principal pathways located in the present work are described in Table 1. Three distinct ring polymer minima were identified. The first, denoted min1, corresponds to localisation of all the beads in one permutation-inversion isomer of the dimer minimum. Four transition states were identified for degenerate rearrangements of this minimum, associated with acceptor tunneling, bifurcation, and donor-acceptor interchange via $C_{i}$ or $C_{2}$ transition states. Two additional paths were characterised connecting the localised minimum with minima exhibiting delocalised acceptor protons (min1), and a delocalised donor proton (min3) (Table 1). Snapshots along these paths are illustrated in Figure 1, and disconnectivity graphs at selected values of $\beta$ are shown in Figure 2. The energy profiles as a function of $\beta$ are collected in Figure 3, which illustrates how the pathways become flatter in the region of the transition state as $\beta$ increases and the magnitude of $\lambda_{\text {neg }}$ decreases. This flat region enables minimization algorithms with an early stopping condition to provide a reasonable guess for instanton pathways if applied with due caution. ${ }^{62,63}$ For $P=201$ the quantum to classical transition, in terms of localisation for the transition state beads, occurs when $\beta$ falls below

9000, 6000, 11000, 11000 hartree $^{-1}$ for acceptor, bifurcation, and donor-acceptor interchange 
$C_{i}$ and $C_{2}$, respectively. The stationary points and pathways were recalculated at intervals of $\beta=1000$ hartree $^{-1}$.

Table 1: Notation for the transition states and associated minima characterised for the ring polymer landscape of water dimer for the MBPOL potential. Here 'classical minimum' (min1) indicates that all the beads are localised at a permutation-inversion isomer of the minimum on the dimer potential energy surface.

\begin{tabular}{|l|c|c|}
\hline \multicolumn{1}{|c|}{ first minimum } & transition state & second minimum \\
\hline classical minimum & acceptor & classical minimum \\
classical minimum & donor-acceptor. $C_{i}$ & classical minimum \\
classical minimum & bifurcation & classical minimum \\
classical minimum & donor-acceptor. $C_{2}$ & classical minimum \\
classical minimum & min1 $\leftrightarrow$ min2 & delocalised min2 \\
classical minimum & min1 $\leftrightarrow$ min3 & delocalised min3 \\
classical minimum & rotation & delocalised min3 \\
\hline
\end{tabular}

One additional path linking different permutational isomers of the localised minimum and the minimum with a delocalised donor proton was also located (Supporting Information).

\subsection{Stationary Points on the Ring Polymer Landscape}

Here we show how the eigenvalues and eigenvectors of transition states on the ring polymer landscape can be used to elucidate and categorise fundamental properties of the corresponding rearrangements between alternative permutational isomers. It is these paths that will determine observables that characterise quantum effects, including tunneling and rates.

The number of positive, negative, and zero eigenvalues for each stationary point is the first key feature. In each case there are six zero eigenvalues corresponding to overall translation and rotation, and analytical forms are available for the corresponding eigenvectors. ${ }^{64}$ The eigenvalues for these degrees of freedom can conveniently be shifted by adding a multiple of $e_{i} e_{j}$ to Hessian element $H_{i j}$, where $\mathbf{e}$ is the normalised eigenvector. ${ }^{61}$ This procedure provides a straightforward way to separate the corresponding degrees of freedom.

For the values of $\beta$ and $P$ considered here, the magnitude of $\lambda_{\text {neg }}$ is large enough to define transition states of $U_{P}(\beta, \mathbf{X})$ to high precision. However, most of the non-classical stationary 

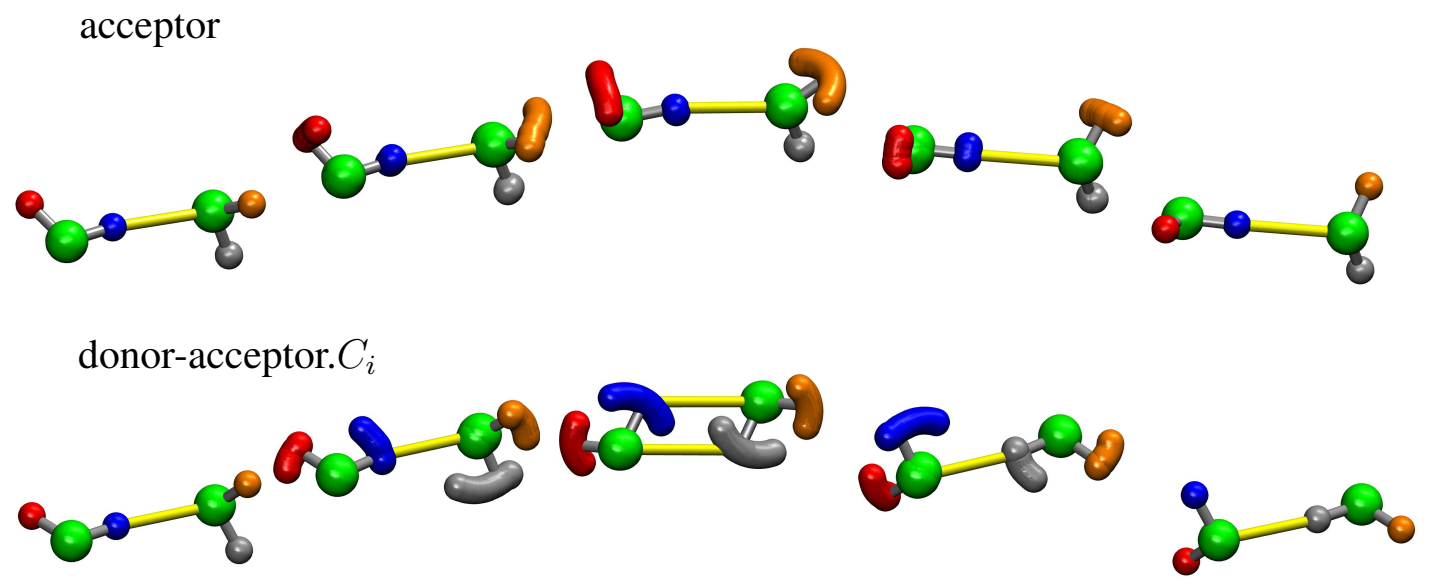

bifurcation
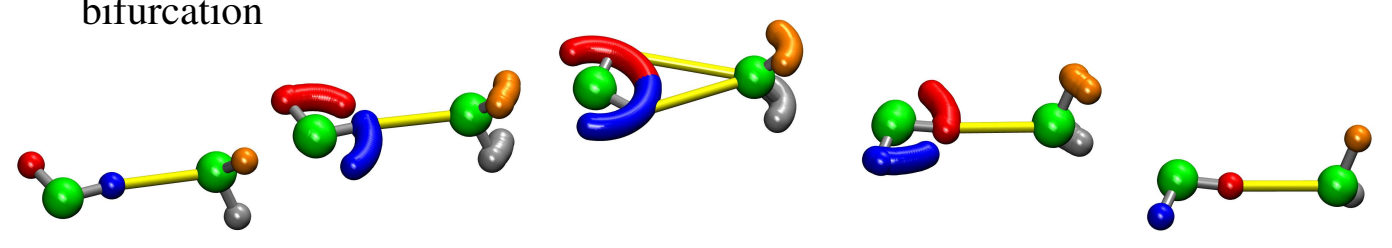

donor-acceptor. $C_{2}$
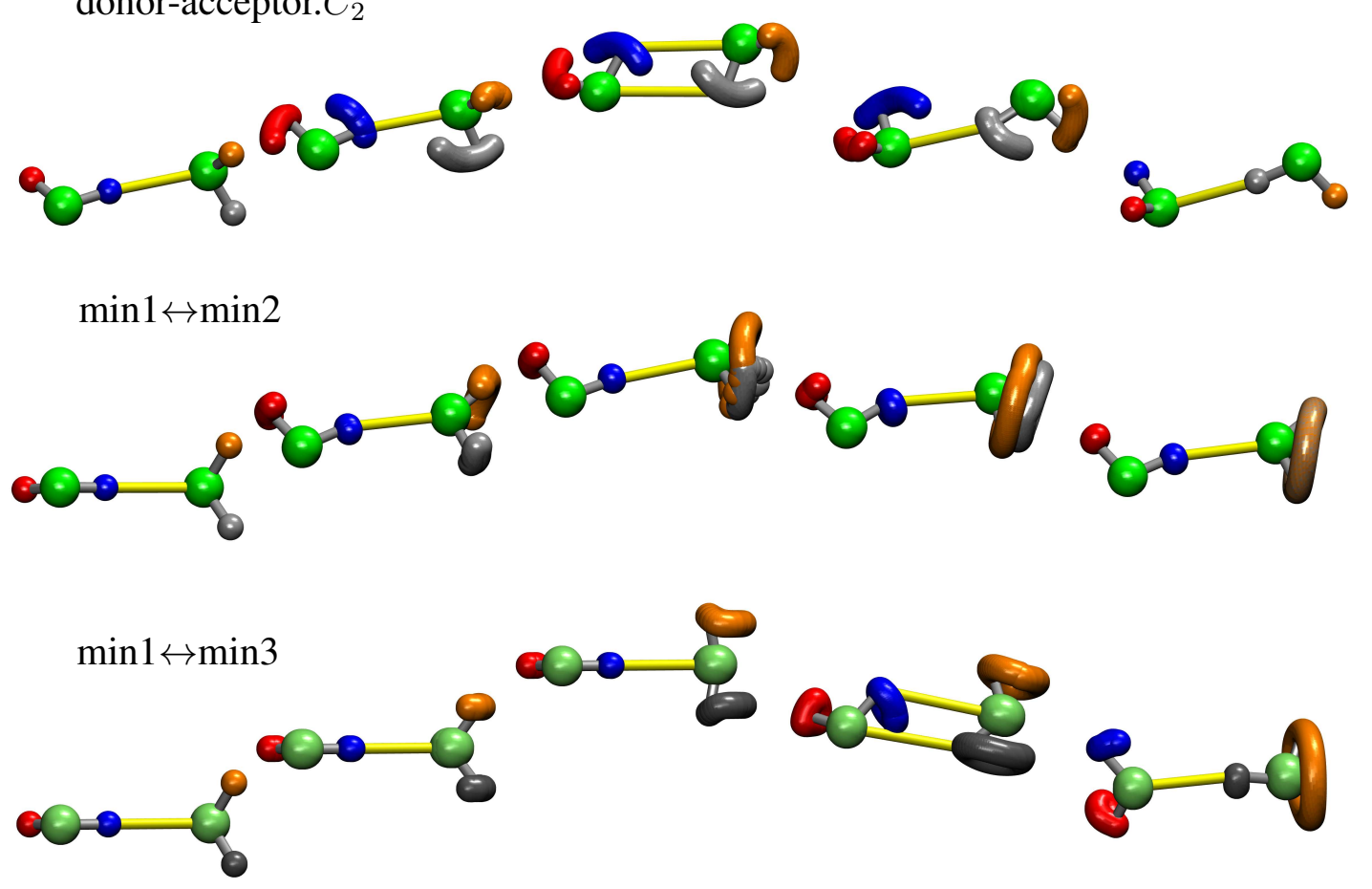

Figure 1: Snapshots of configurations along the pathways for six paths characterised in water dimer (Table 1) described by 201 ring polymer beads for the MBPOL potential at $\beta=20000$ hartree $^{-1}$. Each set includes the two minima, the transition state, and two intervening structures, positioned at relative heights corresponding approximately to the energy. The $\mathrm{O}-\mathrm{H}$ bonds and hydrogen-bonds (yellow) are included for the first bead to guide the eye. 

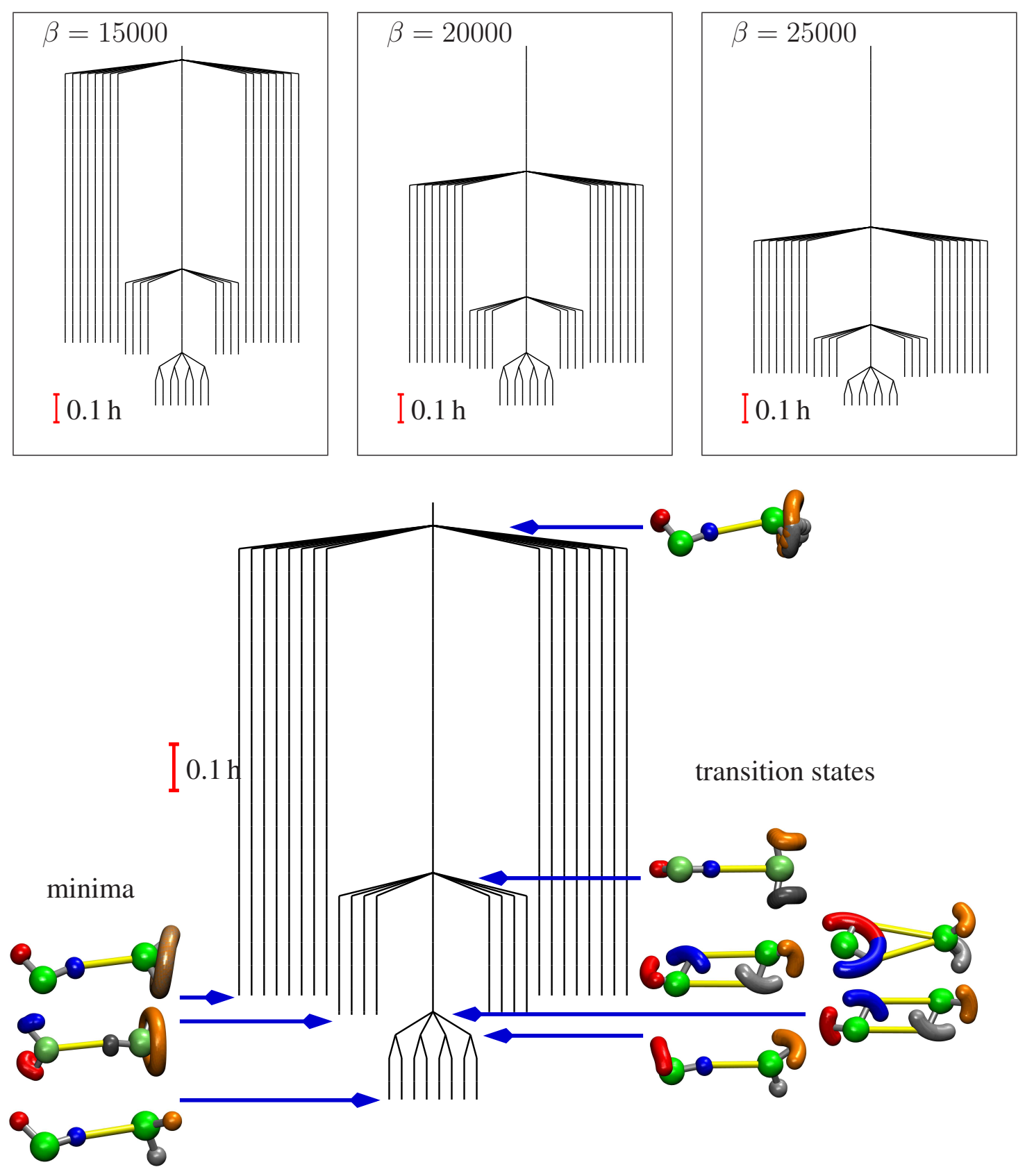

Figure 2: Disconnectivity graphs for the ring polymer landscape of water dimer described by 201 ring polymer beads for the MBPOL potential. The top three panels show how the landscape changes as a function of $\beta$ for values of $1 / k_{B} T$ corresponding to 15000,20000 and 25000 in hartree ${ }^{-1}$. The bottom panel shows a magnification for $\beta=20000$ hartree $^{-1}$ with structures of the ring polymer minima and transition states superimposed. Transition states linking minima that can interconvert via lower energy pathways do not change the structure of the graph, but are included for illustration at appropriate positions on the vertical energy axis. See also Figure 4 for a comparison of classical and path integral landscapes. 

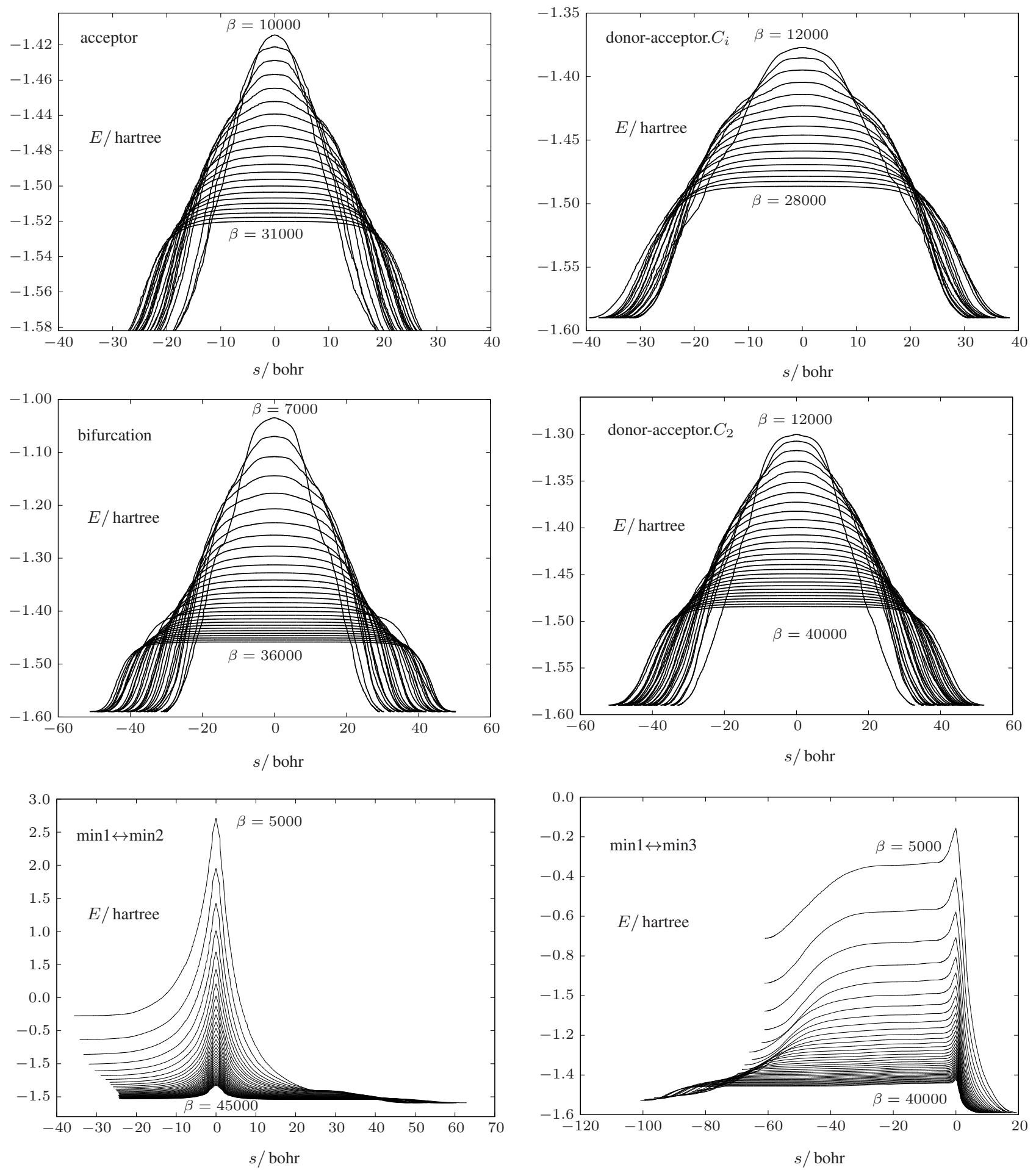

Figure 3: Energy in kcal/mol as a function of integrated path length for the six distinct paths characterised in water dimer (Table 1) described by 201 ring polymer beads for the MBPOL potential. The plots in each panel correspond to $\beta$ values in steps of 1000 hartree $^{-1}$ between the maximum and minimum values, as marked. 
points, where the beads are delocalised away from the classical result with $P=1$, exhibit an additional Hessian eigenvalue close to zero, which arises from the cyclic permutation symmetry for the beads. ${ }^{23} U_{P}(\beta, \mathbf{X})$ is invariant to the transformation $\mathbf{X}^{(\alpha)} \rightarrow \mathbf{X}^{(\alpha+1)}$. In the limit of infinite $P$ this transformation constitutes a continuous symmetry (corresponding to time invariance), which produces a zero Hessian eigenvalue (Noether's theorem). ${ }^{65}$ We denote the corresponding vector of displacements by

$$
\mathbf{v}=\left\{\mathbf{X}^{(2)}-\mathbf{X}^{(1)}, \mathbf{X}^{(3)}-\mathbf{X}^{(2)}, \ldots, \mathbf{X}^{(1)}-\mathbf{X}^{(P)}\right\}
$$

and the normalised vector by $\hat{\mathbf{v}}$. The overlap of $\hat{\mathbf{v}}$ with the eigenvector corresponding to an extra zero eigenvalue after shifting the zeros for overall translation and rotation provides a measure of convergence for this mode with $P$. Similarly, the curvature in this direction, $\hat{\mathbf{v}}^{T} \mathbf{H} \hat{\mathbf{v}}$, should tend to zero as $P$ becomes large. These values are collected in Table 2. The results for the acceptor tunneling rearrangement for three values of $P$ illustrate the convergence of the cyclic permutation mode, and similar values for the overlap and the curvature are obtained with 201 beads for bifurcation tunneling and the two donor-acceptor interchange processes.

We also checked the degeneracies of the Hessian eigenvalues for the minima and transition states with odd and even $P$. For the classical minimum, denoted min1, where all the beads are localised, the double degeneracies expected for a cyclic polyene are indeed observed, with a non-degenerate maximum eigenvalue when $P$ is even. There is no additional zero eigenvalue for these classical minima, because $\mathbf{v}=\mathbf{0}$. The degeneracies are broken for the delocalised stationary points, since the values of $V\left(\mathbf{X}^{(\alpha)}\right)$ depend on $\alpha$, introducing diagonal disorder into the Hessian. The four delocalised transition states connecting permutationinversion isomers of the classical minimum min1 have lower energies than the corresponding classical transition states because the beads are distributed in downhill directions close to the classical steepest-descent pathways. This effect is illustrated in Figure 4, where the low 
Table 2: Properties of the water dimer ring polymer transition states located at $\beta=20000$ hartree $^{-1}$. $U_{P}$ is the energy in hartree and $\lambda_{\text {neg }}$ is the negative eigenvalue in hartree $/ \AA^{2}$. Curvature is the value of $\hat{\mathbf{v}}^{T} \mathbf{H} \hat{\mathbf{v}}$ in hartree $/ \AA^{2}$ for the normalised vector of displacements in the cyclic permutation coordinate, $\hat{\mathbf{v}}$, with $\mathbf{H}$ the Hessian after shifting the eigenvalues corresponding to overall translation and rotation. Overlap is the magnitude of the dot product between $\hat{\mathbf{v}}$ and the eigenvector corresponding to the extra zero eigenvalue (if present). $\mu$ and $\sigma$ are the mean and standard deviation of the displacement between successive beads (in $\AA$ ) in the ring polymer transition state. The standard deviation is much larger for the last two paths, reflecting the much greater variation in bead spacing.

\begin{tabular}{|c|c|c|c|c|c|c|c|}
\hline pathway & $P$ & $U_{P}$ & $\lambda_{\text {neg }}$ & curvature & overlap & $\mu$ & $\sigma$ \\
\hline acceptor & 51 & -0.376286 & -0.0001356 & 0.010314 & 0.9940 & 0.0190 & 0.0356 \\
acceptor & 201 & -1.482827 & -0.0001371 & 0.000657 & 0.9996 & 0.0046 & 0.0075 \\
acceptor & 301 & -2.220541 & -0.0001369 & 0.000293 & 0.9998 & 0.0024 & 0.0033 \\
bifurcation & 201 & -1.353418 & -0.0000241 & 0.001329 & 0.9993 & 0.0138 & 0.0169 \\
donor-acceptor. $C_{i}$ & 201 & -1.446029 & -0.0000931 & 0.000420 & 0.9997 & 0.0093 & 0.0099 \\
donor-acceptor. $C_{2}$ & 201 & -1.382256 & -0.0001627 & 0.000522 & 0.9995 & 0.0104 & 0.0120 \\
min1 $\leftrightarrow \min 2$ & 201 & -0.757402 & -0.5686 & 2.659 & 0.1932 & 0.0924 & 0.2467 \\
$\min 1 \leftrightarrow \min 3$ & 201 & -1.240696 & -0.2407 & 0.06060 & & 0.0484 & 0.0739 \\
\hline
\end{tabular}

energy region of the disconnectivity graphs are compared for the classical landscape and the path integral landscape with $P=201$ and $\beta=25000$ hartree $^{-1}$.

Several transition states were located that connect a permutation-inversion isomer of the classical minimum to delocalised minima, which appear to have no classical analogue. The physical significance of these pathways, if any, is unknown, and we include them for completeness and to illustrate how other features may exist on the path integral landscape. The structure of these transition states is very different from those that link permutationinversion isomers of min1. They have significantly higher energies, and $\lambda_{\text {neg }}$ is three orders of magnitude larger than for the other entries in Table 2. The spacing of the beads is also much less regular, as evidenced by the standard deviation, $\sigma$. Further qualitative differences become evident when the displacements between beads and eigenvector components are visualised, as shown in Figures 5 to 10. The (a) panels in these figures include all the atoms in every image for each transition state, which provides a direct indication of the degree of delocalisation for each atom. However, the structure of the ring polymers is much 
(a)

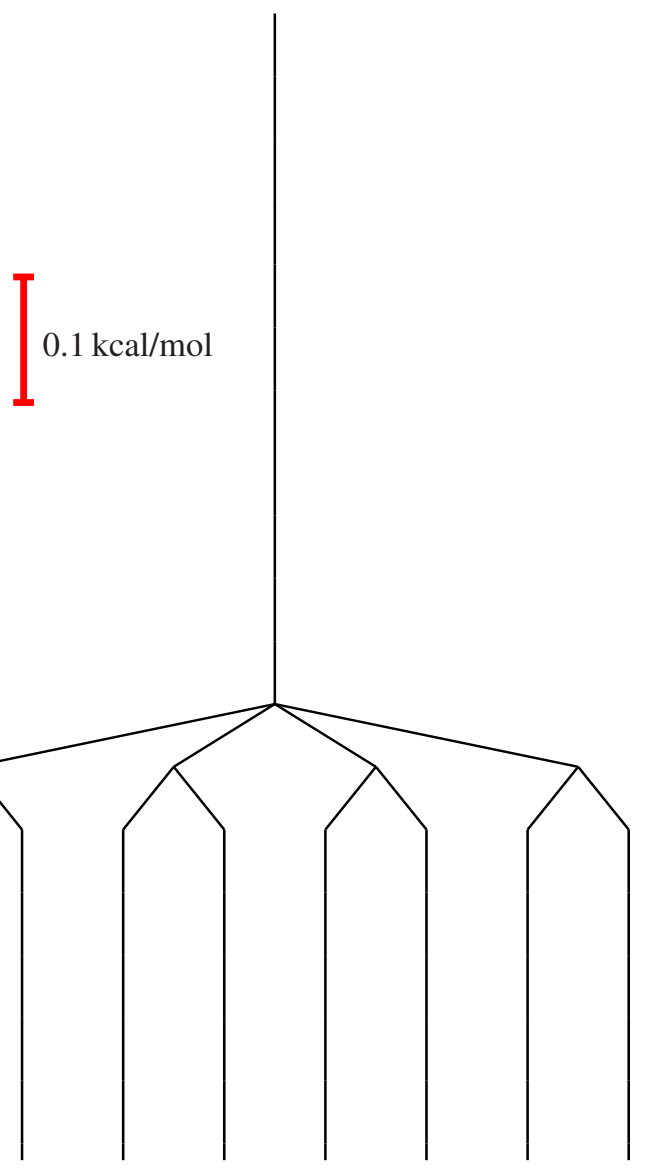

(b)

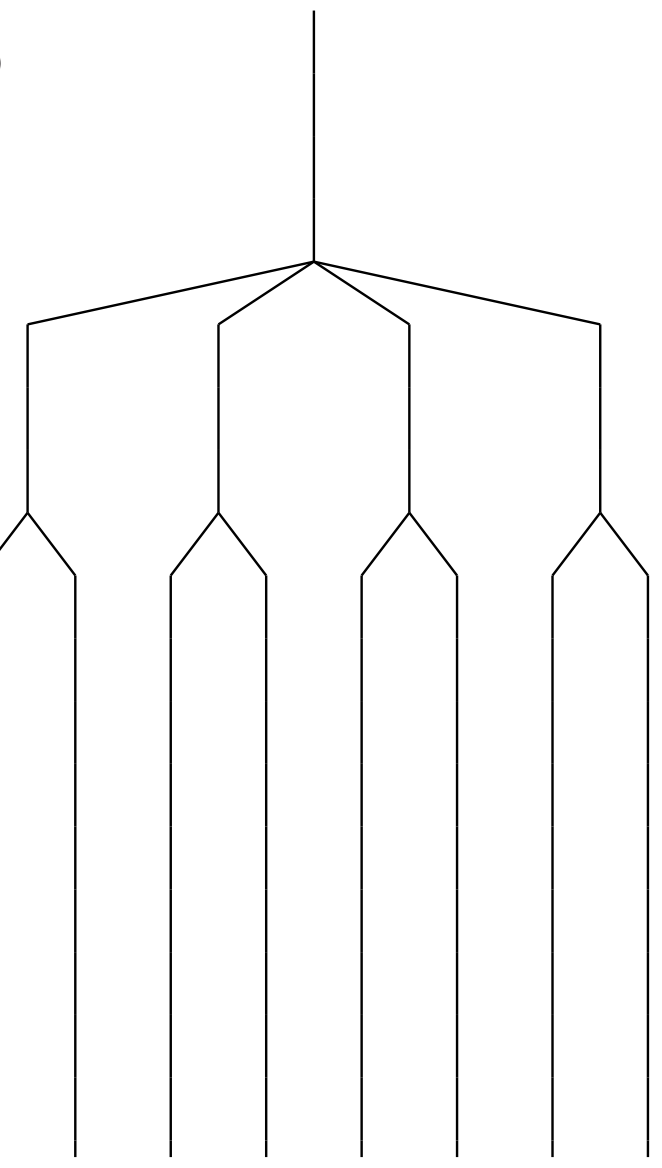

Figure 4: Comparison of disconnectivity graphs for the water dimer including only the classical minima. (a) The ring polymer landscape for 201 beads and $\beta=25000$ hartree $^{-1}$, where the energy has been divided by 201 and converted to kcal/mol for direct comparison with the classical landscape in panel (b). The vertical energy scales are identical. The lower branching in (a) reflects the lower energies of the delocalised transition states for the ring polymer, where beads are distributed close to the classical steepest-descent paths on both sides of the transition state. 
clearer in the (b) panels, where displacement vectors between successive beads are shown. This representation reveals that the two branches of the polymer between the extrema are practically coincident for the acceptor, bifurcation and donor-acceptor interchange paths. A small displacement is introduced in the figures to prevent vectors in the two branches from overlapping. However, for the $\min 1 \leftrightarrow \min 2$ and $\min 1 \leftrightarrow \min 2$ transition states in Figures 9 and 10 the polymers form loops for the delocalised protons, with irregular spacings.

The components of the eigenvector corresponding to $\lambda_{\text {neg }}$ are shown in the (c) panels. For the acceptor, bifurcation and donor-acceptor interchange paths this eigenvector has a clear interpretation, with components parallel to the displacement between beads in one branch, and antiparallel in the other. This structure means that the atoms move in phase along the pathway towards one or other of the two endpoint minima. Hence the eigenvector corresponding to $\lambda_{\text {neg }}$ follows the pattern for the classical transition state, where displacements along the two steepest-descent paths lead to the two distinct minima. For the min1 $\leftrightarrow \min 2$ and $\min 1 \leftrightarrow \min 3$ transition states (and the transition state for the path shown in the SI) the structure of these eigenvectors does not have a clear interpretation in terms of a classical pathway.

The components of the eigenvector for the additional zero eigenvalue in the acceptor, bifurcation and donor-acceptor interchange paths in the (d) panels match the displacements between beads in the (b) panels. These eigenvectors correspond to the cyclic permutation mode $\hat{\mathbf{v}}$ defined above, and the magnitude of the dot product between the normalised eigenvector and $\hat{\mathbf{v}}$ is close to unity (Table 2 ), even for $P=51$ in the acceptor mode. The curvatures for $\hat{\mathbf{v}}$ are orders of magnitude larger than the additional zero Hessian eigenvalue, because there is still some residual mixing with other degrees of freedom for these finite values of $P$.

The $\min 1 \leftrightarrow \min 2$ and $\min 1 \leftrightarrow \min 3$ transition states are again qualitatively different, probably because of the irregular spacing between the beads. There is some similarity between the eigenvector components in Figure 9 and the displacements between beads, but it breaks down where the beads are far apart. The $\min 1 \leftrightarrow \min 3$ transition state lacks an additional 
(a)

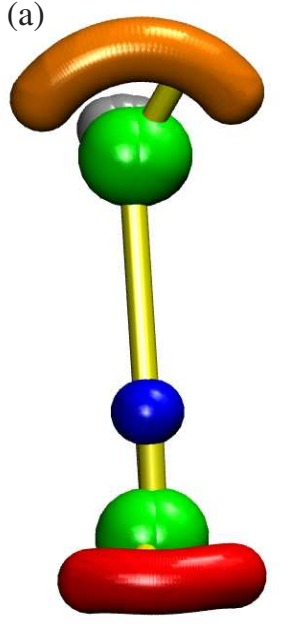

(b)
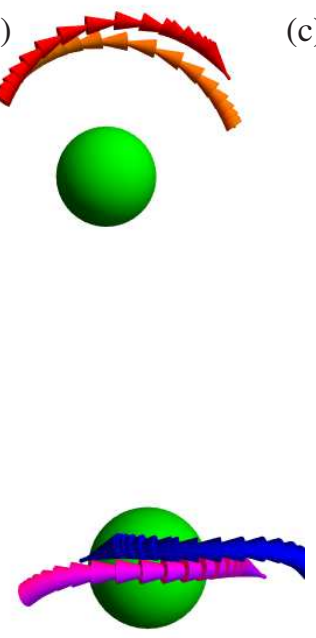

(c)

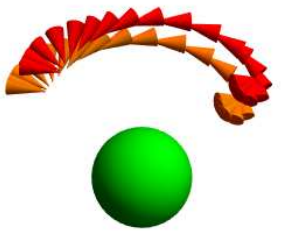

(d)
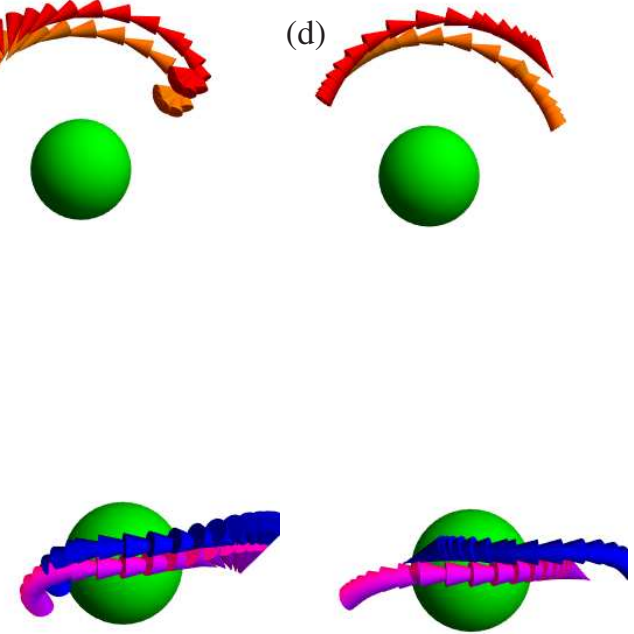

Figure 5: Analysis of the transition state for acceptor tunneling with 201 beads at $\beta=$ 20000 hartree $^{-1}$. (a) Transition state with all atoms included for the 201 images. The four protons are coloured silver, orange, red and blue, and the oxygens are show in green to match the snapshots in Figures 1 and 2. (b) Vectors corresponding to the displacement of two of the hydrogen atoms between successive images for magnitudes exceeding $10^{-6}$ after normalisation. The vectors are coloured differently and shifted by $0.1 \AA$ for the two parts of the ring polymer defined by the extrema. For the first proton the colours are blue and magenta, and for the second proton they are red and orange. Magenta and orange correspond to one part of the ring, and blue and red to the other. (c) Vectors corresponding to the components of the unique Hessian eigenvector with the negative eigenvalue, plotted at the atom sites, with the same colour scheme and shift as part (b). (d) Vectors corresponding to the components of the Hessian eigenvector corresponding to the additional zero eigenvalue, plotted at the atom sites, with the same colour scheme as part (b). In parts (c) and (d) a cutoff of $2 \times 10^{-6}$ was applied for the magnitude of the normalised eigenvector component. For clarity, every 5th displacement or eigenvector component above the cutoff is shown in parts (b) to (d). The vectors corresponding to the extra zero eigenvalue in (d) are parallel to the displacements between the beads in (b), while the phase is reversed for one part of the polymer in part (c), corresponding to motion along the rearrangement pathway. 
(a)

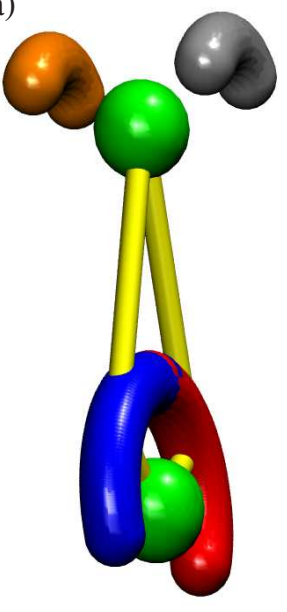

(b)
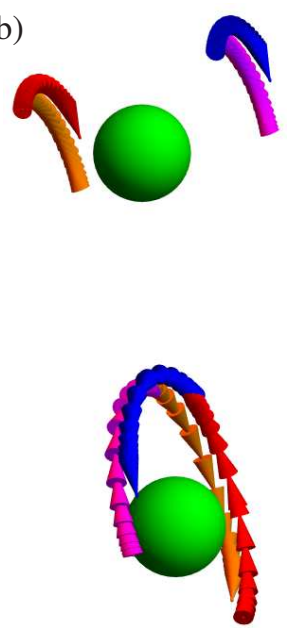

(c)

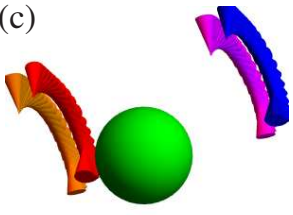

(d)
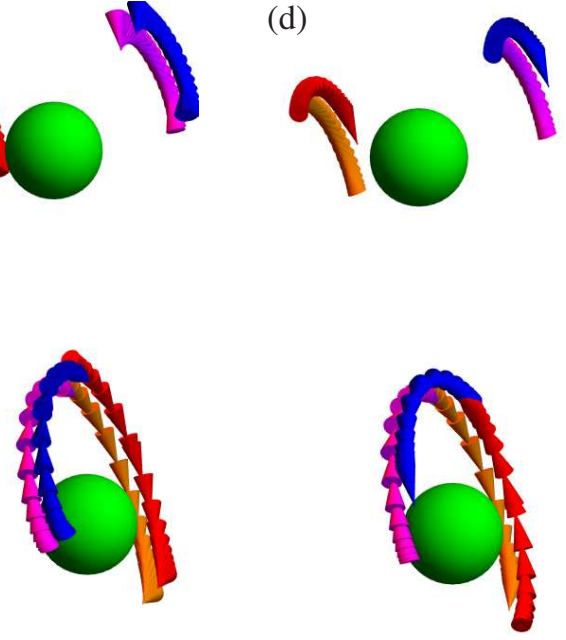

Figure 6: Analysis of the transition state for bifurcation tunneling with 201 beads at $\beta=20000$ hartree $^{-1}$. (a) Transition state with all atoms included for the 201 images. Colour schemes as for Figure 5. (b) Vectors corresponding to the displacement of the four hydrogen atoms between successive images for magnitudes exceeding $10^{-5}$ after normalisation. A shift of by $0.1 \AA$ was added for clarity to separate the two parts of the polymer. (c) Vectors corresponding to the components of the unique Hessian eigenvector with the negative eigenvalue, plotted at the atom sites, with the same colour scheme and shift as part (b). (d) Vectors corresponding to the components of the Hessian eigenvector corresponding to the additional zero eigenvalue, plotted at the atom sites, with the same colour scheme and shift as part (b). In parts (c) and (d) a cutoff of $7.5 \times 10^{-6}$ was applied for the magnitude of the eigenvector component. For clarity, every 4th displacement or eigenvector component above the cutoff is shown in parts (b) to (d). The eigenvectors follow the same pattern as for acceptor tunneling in Figure 5. 


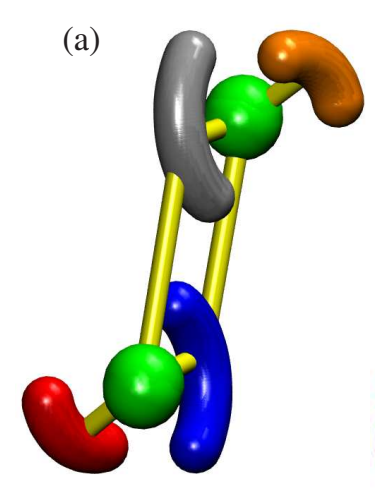

(b)

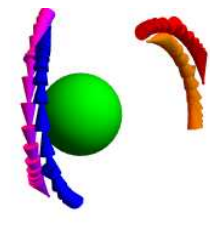

(c)

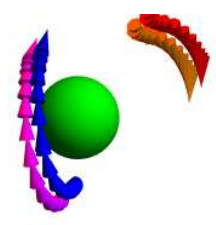

(d)
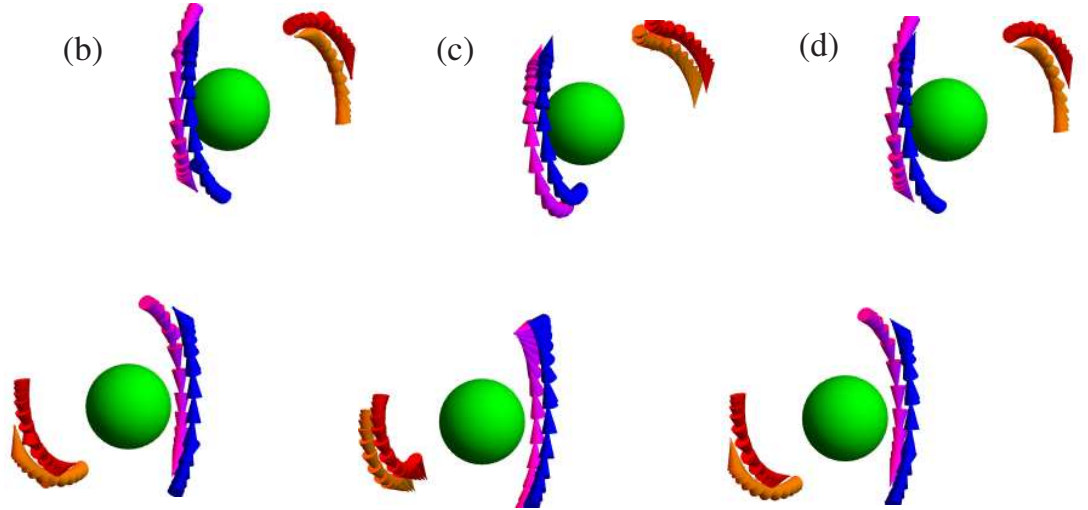
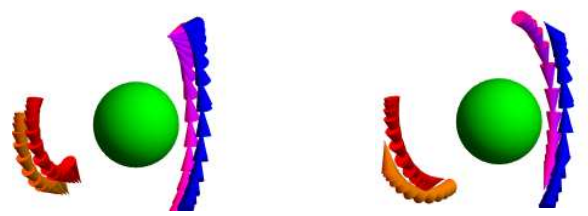

Figure 7: Analysis of the transition state for donor-acceptor. $C_{i}$ tunneling with 201 beads at $\beta=20000$ hartree $^{-1}$. (a) Transition state with all atoms included for the 201 images. Colour schemes as for Figure 5. (b) Vectors corresponding to the displacement of the four hydrogen atoms between successive images for magnitudes exceeding $10^{-7}$ after normalisation. (c) Vectors corresponding to the components of the unique Hessian eigenvector with the negative eigenvalue, plotted at the atom sites. (d) Vectors corresponding to the components of the Hessian eigenvector corresponding to the additional zero eigenvalue, plotted at the atom sites. In parts (c) and (d) a cutoff of $4 \times 10^{-5}$ was applied for the magnitude of the eigenvector component. For clarity, every 6th displacement or eigenvector component above the cutoff is shown in parts (b) to (d). The eigenvectors follow the same pattern as for acceptor tunneling in Figure 5. 


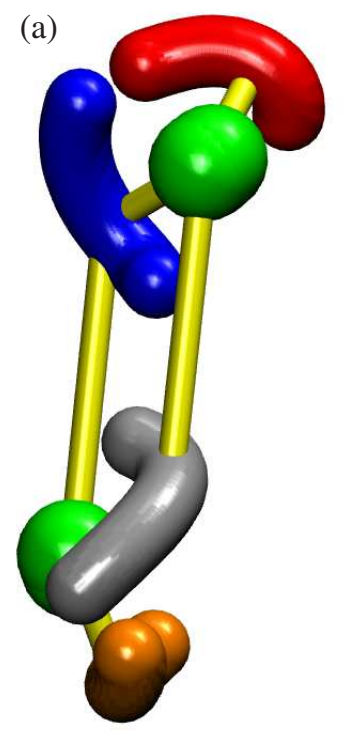

(b)
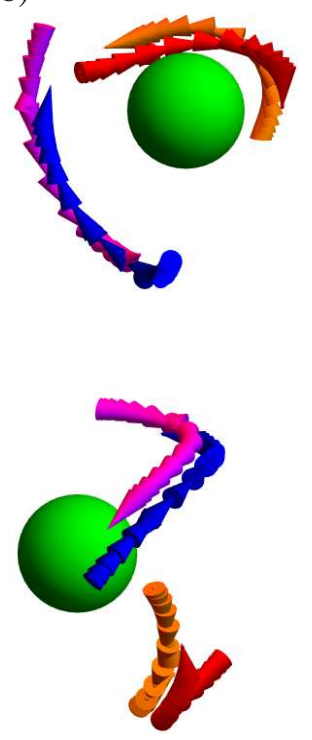

(c)
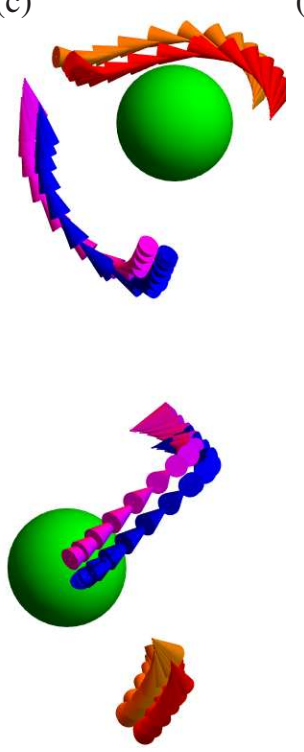

(d)
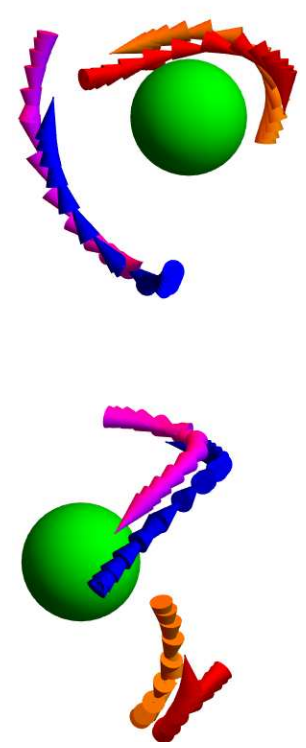

Figure 8: Analysis of the transition state for donor-acceptor. $C_{2}$ tunneling with 201 beads at $\beta=20000$ hartree $^{-1}$. (a) Transition state with all atoms included for the 201 images. Colour schemes as for Figure 5. (b) Vectors corresponding to the displacement of the four hydrogen atoms between successive images for magnitudes exceeding $10^{-5}$ after normalisation. In this case red and blue correspond to the two different acceptor protons. (c) Vectors corresponding to the components of the unique Hessian eigenvector with the negative eigenvalue, plotted at the atom sites. (d) Vectors corresponding to the components of the Hessian eigenvector corresponding to the additional zero eigenvalue, plotted at the atom sites. In parts (c) and (d) cutoffs of $2 \times 10^{-3}$ and $10^{-7}$ were applied for the magnitude of the eigenvector component. Every displacement or eigenvector component above the cutoff is shown in parts (b) to (d). The eigenvectors follow the same pattern as for acceptor tunneling in Figure 5. 

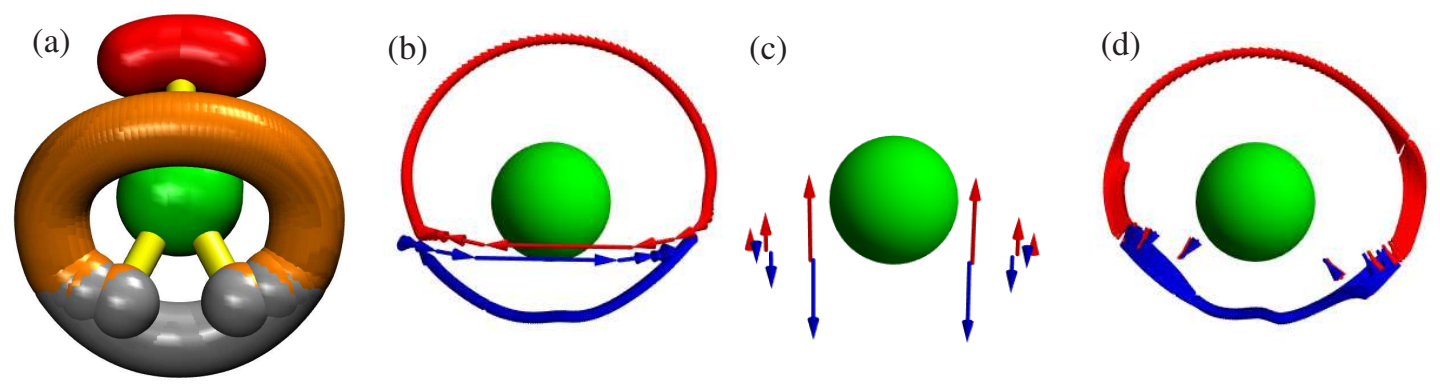

Figure 9: Analysis of the transition state connecting min1 and min2 with 201 beads at $\beta=20000$ hartree $^{-1}$. (a) Transition state with all atoms included for the 201 images. Colour schemes as for Figure 5. (b) Vectors corresponding to the displacement of two of the hydrogen atoms between successive images for magnitudes exceeding $10^{-5}$ after normalisation. In this case red and blue correspond to the two different acceptor protons with a shift of $0.05 \AA$ for the red displacements to prevent the arrows from overlapping. (c) Vectors corresponding to the components of the unique Hessian eigenvector with the negative eigenvalue, plotted at the atom sites. This eigenvector is mainly localised on a small number of beads. (d) Vectors corresponding to the components of the Hessian eigenvector corresponding to the additional zero eigenvalue. In parts (c) and (d) cutoffs of $2 \times 10^{-3}$ and $10^{-7}$ were applied for the magnitude of the eigenvector component. Every displacement or eigenvector component above the cutoff is shown in parts (b) to (d). In (d) the components follow the pattern of displacements for the beads in part (a) where the images are close together, but rotate away from this path where the separation is large. The colours in parts (c) and (d) correspond to the two acceptor protons, as in part (b). 
(a)

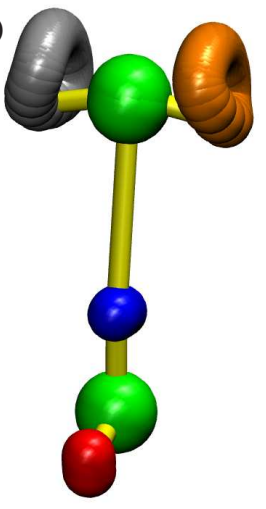

(b)

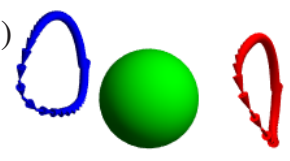

(c)

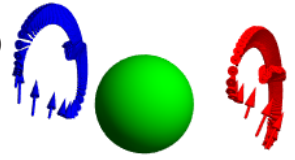

(d)

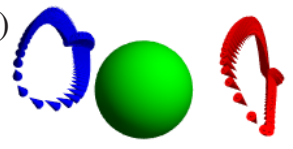

Figure 10: Analysis of the transition state connecting min1 and min3 with 201 beads at $\beta=20000$ hartree $^{-1}$. (a) Transition state with all atoms included for the 201 images. Colour schemes as for Figure 5. (b) Vectors corresponding to the displacement of two of the hydrogen atoms between successive images; all components are included. In this case red and blue correspond to the two different acceptor protons. (c) Vectors corresponding to the components of the unique Hessian eigenvector with the negative eigenvalue, plotted at the atom sites. This eigenvector has larger components where the beads are further apart. (d) Vectors corresponding to the components of the Hessian eigenvector corresponding to the smallest positive Hessian eigenvalue. This transition state does not have an additional zero eigenvalue. In parts (c) and (d) no cutoffs were applied for the magnitude of the eigenvector component; every displacement or component is shown in parts (b) to (d). In (d) the components generally follow the pattern of displacements for the beads in part (a). The colours in parts (c) and (d) correspond to the two acceptor protons, as in part (b). 
zero eigenvalue altogether.
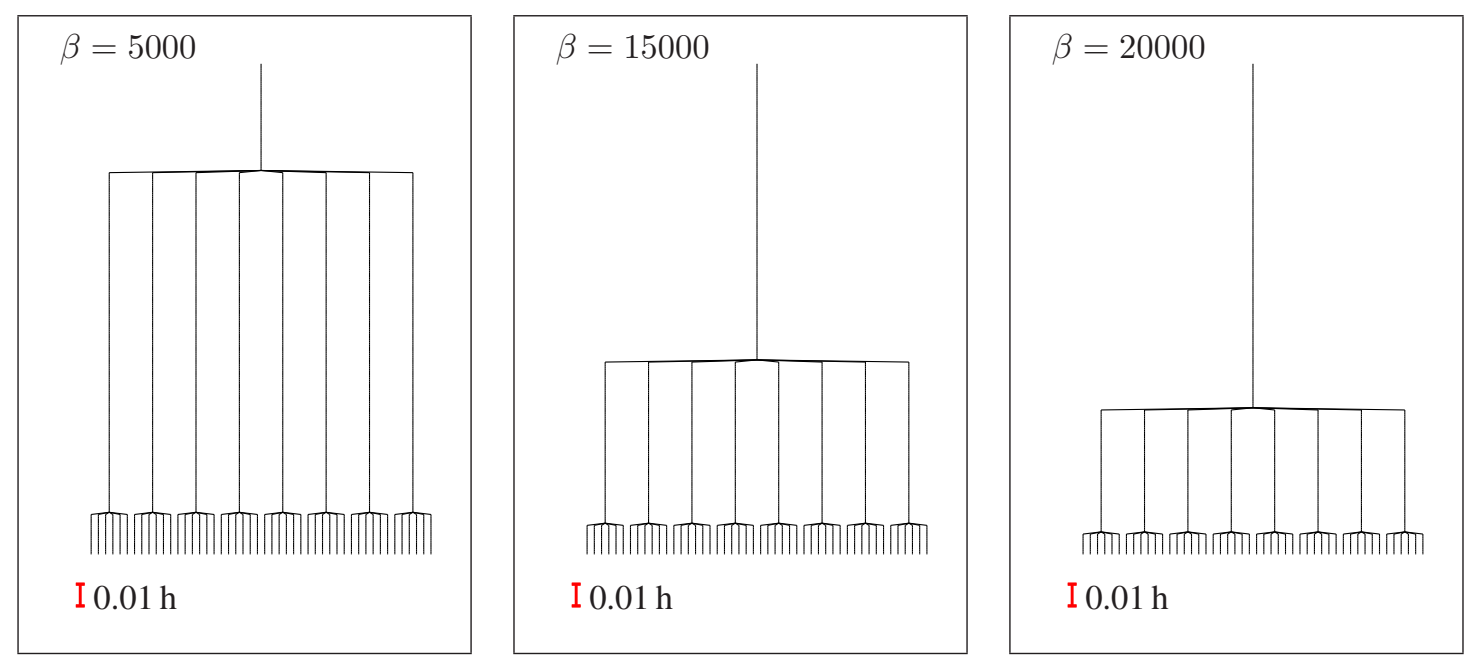

Figure 11: Disconnectivity graphs for the ring polymer landscape of water trimer described by 51 ring polymer beads for the MBPOL potential. The three panels show how the landscape changes as a function of $\beta$ for values of $1 / k_{B} T$ corresponding to 5000, 15000 and 20000 in hartree ${ }^{-1}$ (left to right). At the highest temperature (left) the transition states are the classical, localised structures; they become delocalised for the flip and bifurcation transition states above $\beta=8000 \mathrm{~h}^{-1}$ and $\beta=5000 \mathrm{~h}^{-1}$, respectively. The minima are classical throughout this temperature range.

\section{Discussion}

The ring polymer energy landscape for the water dimer is particularly simple and can be characterized manually. However, most other systems cannot be so easily explored. Even the simple extension of adding a water molecule to the cluster leads to substantially more complicated landscape. Thus, knowledge of the behaviour of delocalised transition states on the path integral energy landscape can be used to automate the procedure for calculating tunneling splittings for larger systems such as the water hexamer, where the relevant tunneling pathways were identified in conjunction with experimental efforts. ${ }^{29}$ To provide examples for larger systems we illustrate the landscape for water trimer and pentamer described by the MBPOL potential and $P=51$ beads in figures 11 and 12 . For the trimer, the low-barrier flip rearrangement connects permutation-inversion isomers in sets of six, and 48 

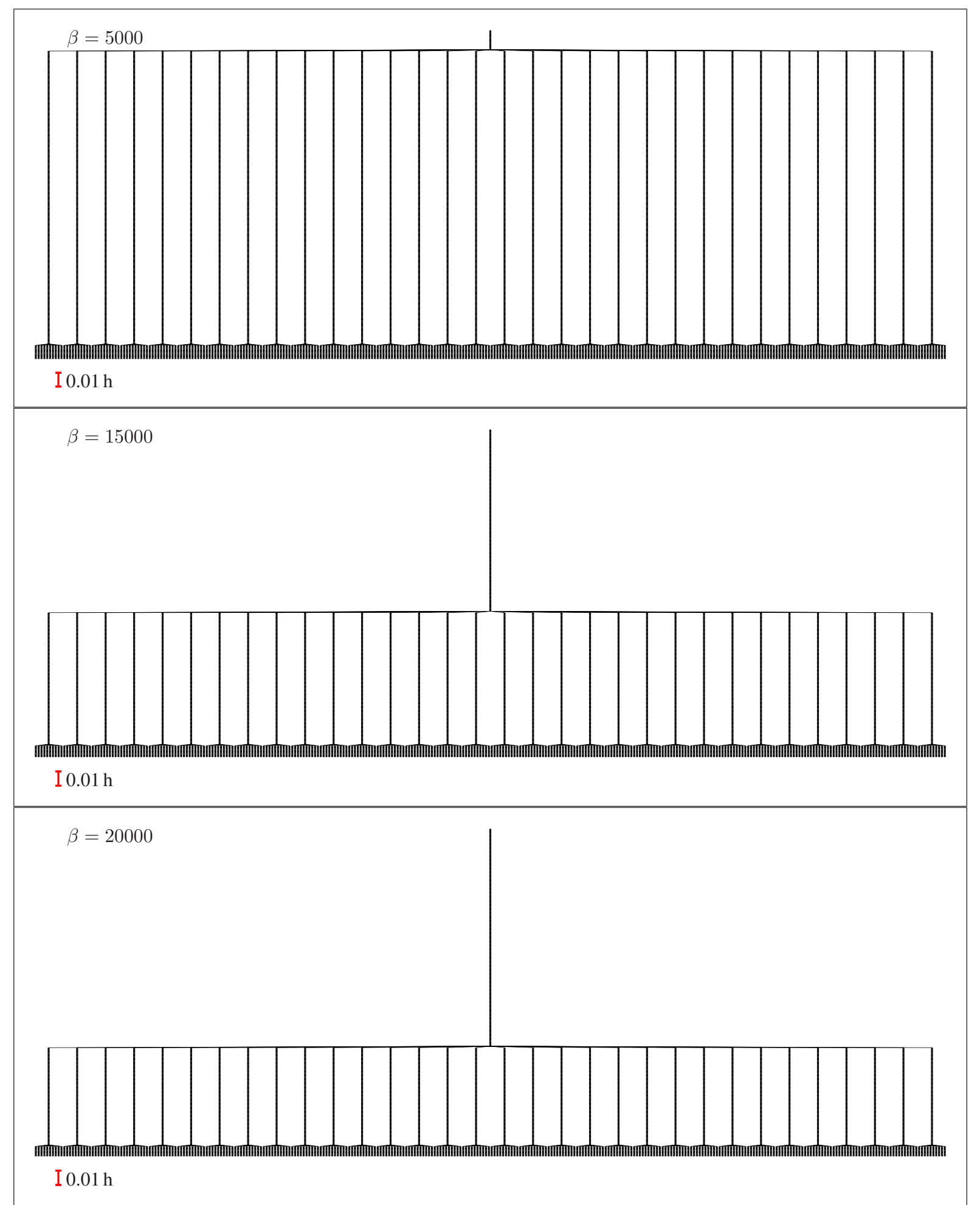

Figure 12: Disconnectivity graphs for the ring polymer landscape of water pentamer described by 51 ring polymer beads for the MBPOL potential with $\beta=5000,15000$ and 20000 hartree $^{-1}$. The transition states become delocalised for the flip and bifurcation above $\beta=10000 \mathrm{~h}^{-1}$ and $\beta=5000 \mathrm{~h}^{-1}$, respectively. The minima are classical throughout this temperature range. 
isomers can interconvert when the bifurcation transition state is included. ${ }^{66}$ The bifurcation mechanism for the MBPOL potential in the classical limit corresponds to majority donor with zero associated flips in the classification scheme of Walsh and Wales. ${ }^{67}$ We expect this pathway to determine the form of the disconnectivity graph, since it probably corresponds to the lowest transition state for the ring polymer. Similarly, the pentamer also supports flip and bifurcation rearrangements, connecting sets of 20 permutation-inversion isomers for the low-barrier mechanism, expanding to 320 isomers when both pathways are feasible. ${ }^{68,69}$ For the MBPOL potential the classical bifurcation pathway corresponds to the A-bif/E-bif process for the classification scheme developed in previous work. ${ }^{70,71}$

Several aspects of the ring polymer landscape are worth discussing in the context of automation. For the more complicated minima and transition states, such as those involved in the $\min 1 \leftrightarrow \min 2$ and $\min 1 \leftrightarrow \min 3$ pathways, the large barriers translate into tunneling processes beyond experimental resolution. The disconnectivity graphs in Figure 2 thus provide a useful guide to filter out either unphysical or irrelevant instantons, which may exist on the ring polymer landscape. Furthermore, the values of the curvature, the mean, and the standard deviation of the displacement between beads provide useful quantities for automated filtering during a transition state search.

The search for individual instantons can also be be automated. Initial guesses can be efficiently generated from knowledge of the classical energy landscape, and projecting out the additional zero eigenvalue corresponding to the cyclic permutation mode can lead to significant speedups when characterising the delocalised transition states of interest. This gain in efficiency, combined with automated tests based on the geometrical properties discussed above, can also be exploited in automatically surveying the ring polymer landscape. In this way, we can avoid full calculations of the tunneling splittings for all the instantons, which would otherwise be a limiting step for large systems such as quantum glasses ${ }^{72,73}$ or atomic clusters. ${ }^{74}$ 


\section{Conclusions}

We have characterized the ring polymer energy landscape for the water dimer. The localised minima were identified, along with the localised (classical) and delocalised (instanton) transition states that connect the individual wells and give rise to tunneling splittings. We also find additional delocalised minima and transition states that are qualitatively different from previously known instantons on the landscape. These new structures are unlikely to contribute significantly to the tunneling spectrum due to the very high TS energies leading to undetectably small tunneling splittings (although the structures may be contributions from higher rotational states ${ }^{75}$ ). For ground-state tunneling splittings these structures could probably be filtered out automatically.

We provide a detailed analysis of the eigenvectors for zero eigenvalue modes that arise due to the permutational invariance of the ring polymer beads. By projecting out these modes transition states can be found more efficiently. Furthermore, the geometric analysis of the bead distribution suggests that stationary points of questionable relevance to the tunneling spectrum could be filtered out during an automated search for the instanton structures. Such

automated searches could greatly simplify the task of generating relevant tunneling spectra for poorly understood or large systems.

\section{Acknowledgement}

We gratefully acknowledge financial support from the EPSRC.

\section{Supporting Information Available}

Animations of each path at a specific value of $\beta$. Snapshots of ring polymer structures along the additional 'rotation' pathway. This material is available free of charge via the Internet at http://pubs.acs.org/. 


\section{References}

(1) Liu, K.; Cruzan, J. D.; Saykally, R. J. Water Clusters. Science 1996, 271, 929-932.

(2) Pérez, C.; Muckle, M. T.; Zaleski, D. P.; Seifert, N. A.; Temelso, B. n.; Shields, G. C.; Kisiel, Z.; Pate, B. H. Structures of Cage, Prism, and Book Isomers of Water Hexamer from Broadband Rotational Spectroscopy. Science 2012, 336, 897-901.

(3) Wang, Y.; Bowman, J. M. Towards an ab initio flexible potential for water, and postharmonic quantum vibrational analysis of water clusters. Chem. Phys. Lett. 2010, 491, $1-10$.

(4) Wang, Y.; Bowman, J. M. Ab initio potential and dipole moment surfaces for water. II. Local-monomer calculations of the infrared spectra of water clusters (9 pages). $J$. Chem. Phys. 2011, 134, 154510.

(5) Burnham, C. J.; Li, J.; Xantheas, S. S.; Leslie, M. The parameterization of a Thole-type all-atom polarizable water model from first principles and its application to the study of water clusters $(\mathrm{n}=2-21)$ and the phonon spectrum of ice Ih. J. Chem. Phys. 1999, $110,4566-4581$.

(6) Xantheas, S. S. Ab initio studies of cyclic water clusters (H2O)n (n=1- 6). II. Analysis of many-body interactions. J. Chem. Phys. 1994, 100, 7523-7524.

(7) Xantheas, S. S. Ab initio studies of cyclic water clusters (H2O)n, n=1- 6. III. comparison of density functional theory with MP2 results. J. Chem. Phys. 1995, 102, 4505-4517.

(8) Ceriotti, M.; Fang, W.; Kusalik, P. G.; McKenzie, R. H.; Michaelides, A.; Morales, M. A.; Markland, T. E. Nuclear Quantum Effects in Water and Aqueous Systems: Experiment, Theory, and Current Challenges. Chem. Rev. 2016, 116, 7529.

(9) Ceperley, D. M. Path Integrals in the theory of condensed helium. Rev. Mod. Phys 1995, 67, 279. 
(10) Markland, T. E.; Ceriotti, M. Nuclear quantum effects enter the mainstream. Nat. Rev. Chem. 2018, 2, 0109.

(11) Craig, I. R.; Manolopoulos, D. E. Quantum statistics and classical mechanics: Real time correlation functions from ring polymer molecular dynamics. J. Chem. Phys. 2004, 121, 3368.

(12) Craig, I. R.; Manolopoulos, D. E. Chemical reaction rates from ring polymer molecular dynamics. J. Chem. Phys. 2005, 122, 084106.

(13) Craig, I. R.; Manolopoulos, D. E. A refined ring polymer molecular dynamics theory of chemical reaction rates. J. Chem. Phys. 2005, 123, 034102.

(14) Cao, J.; Voth, G. A. The formulation of quantum statistical mechanics based on the Feynman path centroid density. I. Equilibrium properties. J. Chem. Phys. 1993, 100, 5093.

(15) Cao, J.; Voth, G. A. The formulation of quantum statistical mechanics based on the Feynman path centroid density. II. Dynamical properties. J. Chem. Phys. 1994, 100, 5106.

(16) Liu, J. Path integral Liouville dynamics for thermal equilibrium systems. J. Chem. Phys. 2014, 140, 224107.

(17) Liu, J.; Zhang, Z. Path integral Liouville dynamics: Applications to infrared spectra of OH, water, ammonia, and methane. J. Chem. Phys. 2016, 144, 034307.

(18) Hele, T. J. H.; Willatt, M. J.; Muolo, A.; Althorpe, S. C. Boltzmann-conserving classical dynamics in quantum time-correlation functions: Matsubara dynamics. J. Chem. Phys. 2015, 142, 134103.

(19) Hele, T. J. H.; Willatt, M. J.; Muolo, A.; Althorpe, S. C. Communication: Relation of 
centroid molecular dynamics and ring-polymer molecular dynamics to exact quantum dynamics. J. Chem. Phys. 2015, 142, 191101.

(20) Habershon, S.; Fanourgakis, G. S.; Manolopoulos, D. E. Comparison of path integral molecular dynamics methods for the infrared absorption spectrum of liquid water. $J$. Chem. Phys. 2008, 129, 074501.

(21) Willatt, M. J.; Ceriotti, M.; Althorpe, S. C. Approximating Matsubara dynamics using the planetary model: Tests on liquid water and ice. J. Chem. Phys. 2018, 148, 102336.

(22) Liu, X.; Liu, J. Critical role of quantum dynamical effects in the Raman spectroscopy of liquid water. Mol. Phys. 2018, 116, 755.

(23) Richardson, J. O.; Althorpe, S. C. Ring-polymer instanton method for calculating tunneling splittings. J. Chem. Phys. 2011, 134, 054109.

(24) Mil'nikov, G. V.; Nakamura, H. Practical implementation of the instanton theory for the ground-state tunneling splitting. J. Chem. Phys. 2001, 115, 6881.

(25) Mil'nikov, G. V.; Nakamura, H. Tunneling splitting and decay of metastable states in polyatomic molecules: invariant instanton theory. J. Chem. Phys. 2008, 10, 1374.

(26) Siebrand, W.; Smedarchina, Z.; Zgierski, M. Z.; Fernandez-Ramos, A. Proton tunnelling in polyatomic molecules: A direct-dynamics instanton approach. Int. Rev. Phys. Chem. $1999,18,5$.

(27) Richardson, J. O.; Althorpe, S. C. Ring-polymer instanton method for calculating tunneling splittings (11 pages). J. Chem. Phys. 2011, 134, 054109.

(28) Richardson, J. O.; Althorpe, S. C.; Wales, D. J. Instanton calculations of tunneling splittings for water dimer and trimer (12 pages). J. Chem. Phys. 2011, 135, 124109. 
(29) Richardson, J. O.; Pérez, C.; Lobsiger, S.; Reid, A. A.; Temelso, B.; Shields, G. C.; Kisiel, Z.; Wales, D. J.; Pate, B. H.; Althorpe, S. C. Concerted hydrogen-bond breaking by quantum tunneling in the water hexamer prism. Science 2016, 351, 1310.

(30) Richardson, J. O. Derivation of instanton rate theory from first principles. J. Chem. Phys. 2016, 144, 114106.

(31) Mátyus, E.; Wales, D. J.; Althorpe, S. C. Quantum tunneling splittings from pathintegral molecular dynamics. J. Chem. Phys. 2016, $144,114108$.

(32) Matyus, E.; Althorpe, S. C. Calculating splittings between energy levels of different symmetry using path-integral methods. J. Chem. Phys. 2016, 144, 114109.

(33) Vaillant, C. L.; Wales, D. J.; Althorpe, S. C. Tunneling-splittings from path-integral molecular dynamics using a Langevin thermostat. J. Chem. Phys. 2018, 148, 234102.

(34) Noé, F.; Fischer, S. Transition networks for modeling the kinetics of conformational change in macromolecules. Curr. Op. Struct. Biol. 2008, 18, 154-162.

(35) Prada-Gracia, D.; Gómez-Gardenes, J.; Echenique, P.; Fernando, F. Exploring the Free Energy Landscape: From Dynamics to Networks and Back. PLoS Comput. Biol. 2009, 5, e1000415.

(36) Wales, D. J. Energy Landscapes: Some New Horizons. Curr. Op. Struct. Biol. 2010, 20, 3-10.

(37) Babin, V.; Leforestier, C.; Paesani, F. Development of a "First Principles" Water Potential with Flexible Monomers: Dimer Potential Energy Surface, VRT Spectrum, and Second Virial Coefficient. J. Chem. Theory Comput. 2013, 9, 5395.

(38) Dyke, T. R. J. Chem. Phys. 1977, 66, 492.

(39) Coudert, L. H.; Hougen, J. T. J. Mol. Spectr. 1988, 130, 86-119. 
(40) Althorpe, S. C.; Clary, D. C. Calculation of the intermolecular bound states for water dimer. J. Chem. Phys. 1994, 101, 3603-3609.

(41) Althorpe, S. C.; Clary, D. C. A new method for calculating the rovibrational states of polyatomics with application to water dimer. J. Chem. Phys. 1995, 102, 4390-4399.

(42) Chen, H.; Liu, S.; Light, J. C. Six-dimensional quantum calculation of the intermolecular bound states for water dimer. J. Chem. Phys. 1999, 110, 168-176.

(43) Huang, X.; Braams, B. J.; Bowman, J. M.; Kelly, R. E. A.; Tennyson, J.; Groenenboom, G. C.; van der Avoird, A. New ab initio potential energy surface and the vibration-rotation-tunneling levels of (H2O)2 and (D2O)2. J. Chem. Phys. 2008, 128, 034312.

(44) Leforestier, C.; Gatti, F.; Fellers, R. S.; Saykally, R. J. Determination of a flexible (12D) water dimer potential via direct inversion of spectroscopic data. J. Chem. Phys. 2002, $117,8710-8722$.

(45) Watanabe, Y.; Taketsugu, T.; Wales, D. J. An ab initio study of tunneling splittings in the water dimer. J. Chem. Phys. 2004, 120, 5993-5999.

(46) Taketsugu, T.; Wales, D. J. Theoretical study of rearrangements in water dimer and trimer. Mol. Phys. 2002, 100, 2793-2806.

(47) Trygubenko, S. A.; Wales, D. J. A Doubly Nudged Elastic Band Method for Finding Transition States. J. Chem. Phys. 2004, 120, 2082-2094.

(48) Henkelman, G.; Jónsson, H. Improved tangent estimate in the nudged elastic band method for finding minimum energy paths and saddle points. J. Chem. Phys. 2000, 113, 9978-9985.

(49) Henkelman, G.; Uberuaga, B. P.; Jónsson, H. A climbing image nudged elastic band 
method for finding saddle points and minimum energy paths. J. Chem. Phys. 2000, 113, 9901-9904.

(50) Munro, L. J.; Wales, D. J. Defect migration in crystalline silicon. Phys. Rev. B 1999, 59, 3969-3980.

(51) Henkelman, G.; Jónsson, H. A dimer method for finding saddle points on high dimensional potential surfaces using only first derivatives. J. Chem. Phys. 1999, 111, $7010-7022$.

(52) Kumeda, Y.; Munro, L. J.; Wales, D. J. Transition States and Rearrangement Mechanisms from Hybrid Eigenvector-Following and Density Functional Theory. Application to $\mathrm{C}_{10} \mathrm{H}_{10}$ and Defect Migration in Crystalline Silicon. Chem. Phys. Lett. 2001, 341, $185-194$.

(53) Murrell, J. N.; Laidler, K. J. Symmetries of activated complexes. Trans. Faraday Soc. 1968, 64, 371-377.

(54) Broyden, C. G. The Convergence of a Class of Double-rank Minimization Algorithms 1. General Considerations. J. Inst. Math. Appl. 1970, 6, 76-90.

(55) Fletcher, R. A new approach to variable metric algorithms. Comput. J. 1970, 13, 317322.

(56) Goldfarb, D. A family of variable-metric methods derived by variational means. Math. Comput. 1970, 24, 23-26.

(57) Shanno, D. F. Conditioning of quasi-Newton methods for function minimization. Math. Comput. 1970, 24, 647-656.

(58) Becker, O. M.; Karplus, M. The topology of multidimensional potential energy surfaces: Theory and application to peptide structure and kinetics. J. Chem. Phys. 1997, 106, $1495-1517$. 
(59) Wales, D. J.; Miller, M. A.; Walsh, T. R. Archetypal energy landscapes. Nature 1998, $394,758-760$.

(60) Uppenbrink, J.; Wales, D. J. When do gradient optimizations converge to saddlepoints? Chem. Phys. Lett. 1992, 190, 447-452.

(61) Wales, D. J. Energy Landscapes; Cambridge University Press: Cambridge, 2003.

(62) Cvitas, M. T.; Althorpe, S. C. Locating Instantons in Calculations of Tunneling Splittings: The Test Case of Malonaldehyde. J. Chem. Theory. Comput. 2016, 12, 787.

(63) Cvitas, M. T. Quadratic String Method for Locating Instantons in Tunneling Splitting Calculations. J. Chem. Theory. Comput. 2018,

(64) Page, M.; McIver, J. W. ? J. Chem. Phys. 1988, 88, 922.

(65) Noether, E. Nachr. Ges. Wiss. Göttingen, Math.-Phys. 1918, K1, 235.

(66) Keutsch, F. N.; Saykally, R. J.; Wales, D. J. Bifurcation tunneling dynamics in the water trimer. J. Chem. Phys. 2002, 117, 8823-8835.

(67) Walsh, T. R.; Wales, D. J. Rearrangements of the water trimer. J. Chem. Soc. Faraday Trans. 1996, 92, 2505-2517.

(68) Wales, D. J. Structure, dynamics, and thermodynamics of clusters - tales from topographic potential surfaces. Science 1996, 271, 925-929.

(69) Wales, D. J.; Walsh, T. R. Theoretical-study of the water pentamer. J. Chem. Phys. 1996, 105, 6957-6971.

(70) Wales, D. J. In Recent Theoretical and Experimental Advances in Hydrogen-Bonded Clusters; Xantheas, S., Ed.; Kluwer: Dordrecht, in press [available from the Los Alamos preprint server at URL http://xxx.lanl.gov/abs/physics/9810032], 1997. 
(71) Wales, D. J. In Advances in Molecular Vibrations and Collision Dynamics; Bowman, J. M., Bačić, Z., Eds.; JAI Press: Stamford, 1998; Vol. 3; pp 365-396.

(72) Markland, T. E.; Morrone, J. A.; Berne, B. J.; Miyazaki, K.; Rabani, E.; Reichman, D. E. Quantum fluctuations can promote or inhibit glass formation. Nat. Phys. 2011, 7, 134 .

(73) Markland, T. E.; Morrone, J. A.; Miyazaki, K.; Berne, B. J.; Reichman, D. R.; Rabani, E. Theory and simulations of quantum glass forming liquids. J. Chem. Phys. 2012, 136, 074511.

(74) Frantsuzov, P. A.; Meluzzi, D.; Mandelshtam, V. A. Structural Transformations and Melting in Neon Clusters: Quantum versus Classical Mechanics. Phys. Rev. Lett. 2006, 96, 113401.

(75) Vaillant, C. L.; Cvitas, M. T. Rotation-tunneling spectrum of the water dimer from instanton theory. Phys. Chem. Chem. Phys. 2018, 20, 26809. 
Graphical TOC Entry

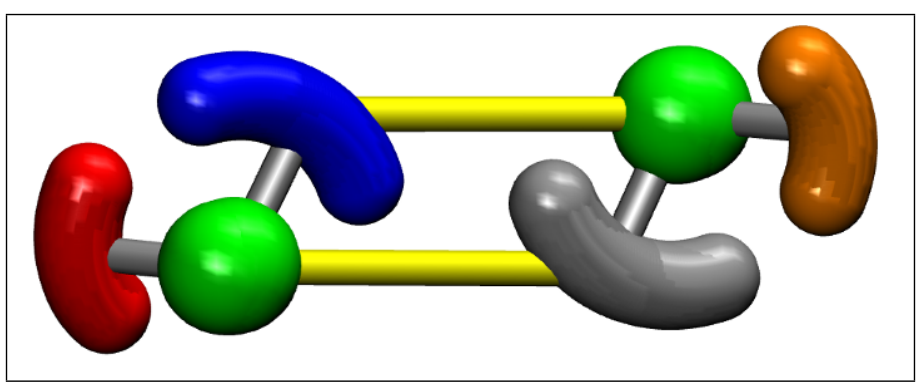

